\title{
Study on Remote Wireless Smart Pot System Based on ZIGBEE+MQTT
}

\author{
Jijun Xing \\ Northwest University of Politics and Law, Xian, Shaanxi, China \\ Xingjijun1@aliyun.com
}

\begin{abstract}
The home internal wireless network smart pot system is established based on the combination of ZigBee and ESP8266. By uploading the plant growth data collected from the system to cloud server of Internet via MQTT protocol, users are allowed to remotely monitor the growing states of plants in the pots by operating mobile APP to water plants, change environment temperature, increase light intensity and kill weeds and insects. This is a scientific breeding solution with low power consumption, low costs and high efficiency.
\end{abstract}

Keywords: Parrot Pat, ZigBee, MQTT, Mobile APP, Remote Monitoring

\section{Introduction}

Nowadays, with the rapid development of wireless network technology, control technology and Internet of Things, the concept of smart home has become increasingly more common. Traditional smart home is realized through wired operation, such as LONWORKS, CEBUS, X-10, RS485, CANBUS, Ethernet, etc. [1]. However, as the wireless network technology is improving day by day, it is possible to conveniently set up the wireless network smart home by taking advantage of ZigBee technology, which not only avoids the trouble in wiring but formulates unified standards for furnishings and meets aesthetic needs [2].

As an element of smart home, smart pot has become more popular than ever among a growing number of young people. Compared with artificial breeding, smart pot can not only bring people a worry-free journey, feeling no difficulty in taking care of plants at home during travels or business trips, but also help to grasp more knowledge about plant breeding and enhance mutual exchanges between human and plants.

This paper puts forward a remote smart pot based on wireless signal transmission. The home internal wireless network smart pot system is established based on the combination of ZigBee and ESP8266. By uploading the diverse plant growth data collected from the system to cloud server of Internet via MQTT protocol, so long as users' smart mobile equipment can access to Internet, they are allowed to remotely monitor the growing states of plants in the pots anywhere. Specifically, after users log in the APP, they can water plants, change environment temperature, increase light intensity and kill weeds and insects in hosting or human monitoring mode. This is a scientific breeding solution with low power consumption, low costs and high efficiency.

\section{ZigBee Technology and MQTT Protocol}

\subsection{ZigBee Technology}

ZigBee refers to a LAN protocol with low power consumption based on IEEE802.15.4, which is an economic and low-power-consumption wireless network communication technology. In accordance with the protocol, ZigBee can carry out communications by 
means of mutual coordination among thousands of tiny sensors. Only with very few energies, these sensors can transmit data by the relay from one sensor to another through radio waves, therefore, they communicate with an extremely high efficiency. [3-4]

ZigBee is allowed to adopt star network, cluster network and mesh network structures [5-6], with one host node controlling up to 254 child nodes and controlled by the network node of upper layer, which forms a large network with up to 65,535 nodes. Besides, it provides three-hierarchy security modes, including no security setting, using Access Control List (ACL) to prevent illegal data acquisition and adopting the symmetric cryptography of Advanced Encryption Standard (AES 128), so as to flexibly determine its security attributes.

\subsection{CC2530 Overview}

The CC2530 is Texas Instrument's second generation ZigBee/IEEE 802.15.4 compliant System-on-Chip with an optimized $8051 \mathrm{MCU}$ core and radio for the $2.4 \mathrm{GHz}$ unlicensed ISM/SRD band [7]. This device enables industrial grade applications by offering state-of-the-art noise immunity, excellent link budget, operation up to 125 degrees and low voltage operation.

Combined with the industry-leading and golden-unit-status Zigbee protocol stack (Z-StackTM)

From Texas Instruments, the CC2530F256 provides a robust and complete Zigbee solution.

In addition, the CC2530 provides extensive hardware support for packet handling, data buffering, burst transmissions, data encryption, data authentication, clear channel assessment, link quality indication and packet timing information.

\subsection{MQTT Protocol}

MQTT refers to a lightweight transmission protocol developed by IBM specifically for Internet of Things, which is designed for lightweight publish/subscribe message transmission, aiming to provide reliable network services for equipment of Internet of Things in the low-bandwidth and unstable network environment. MQTT makes special optimization for networks with low bandwidth and equipment with weak computing power, enabling them to adapt to various application scenarios of Internet of Things. [8-9]

As a protocol designed for the communications of remote sensors and control devices with limited power in massive computations in low-bandwidth and unstable networks, MQTT has the following attributes:

1. Using publish/subscribe message pattern to provide one-to-many message distribution and decoupling of applications

2. Transporting a message that is agnostic to the content of the payload;

3. Using TCP/TP to provide network connectivity;

4. Having three qualities of service for message distribution.

MQTT defines three levels of QoS, which determines how clients and servers ensure message reception. Messages can be published via QoS of any level and client side can also choose QoS of any level to subscribe topics but generally choose the highest level to receive topics.

\section{Introduction to Structure and Hardware and Software Framework of Smart Pot System}

\subsection{Smart Pot System Structure Chart and Description}

System Structure Chart is seen in following Figure 1. 


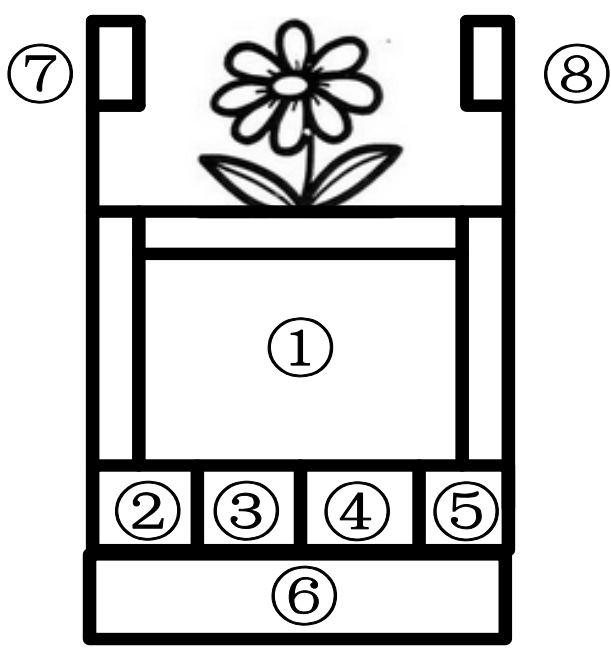

Figure 1. System Structure Chart of Smart Pot

Parts and functions marked in the chart are as follows:

(1) Smart Pot System: consisting of sensor module, wireless network module, function execution module, atomization module and an external ADC module with PCF8591 as core;

(2) Water Slot: feeding water, with one port connected with execution module and the other with the bottom of the pot via metal tube with diameter of $\varnothing 5$, with maximum moisture capacity of $2 \mathrm{~L}$;

(3) Insecticide Slot: storing proportioned insecticide and connected with stepper motor (6), which controls the execution of deinsectization;

(4) Herbicide Slot: storing proportioned herbicide and connected with stepper motor (6), which controls the execution of weeding;

(5) Atomization Slot: containing water, with one port connected with atomization module and the other with stepper motor, which controls the execution of moistening;

(6) Stepper Motor: linear displacement 4-phase stepper motor, connected with (3), (4) and (5) respectively at the bottom of the water slot, with output port connected with (8), controls the execution of weeding, moistening and deinsectization;

(7) 7-1 HD Camera: mobile equipment transmitting HD signal to users, helping users check the maintenance of plants; 7-2 full spectrum light: increasing light of different spectrum to different plants;

(8) Sprayer: with height adjustable, spraying insecticide, herbicide and mist to execute corresponding functions.

\subsection{Hardware Framework}

Hardware framework of smart pot is as follows:

1. Gateway: for initial users, set ESP8266 chip into AP mode, access to it via mobile phone or other mobile equipments, and then set router and user login information. Save settings and then exit. Gateway is successfully set.

2. Sensor Module: the module is composed of a soil moisture sensor HA2001.FDR and an environment temperature sensor DS18B20. The output signal of HA2001.FDR is analog quantity. When using the sensor, its two probes are inserted into the soil of the pot to detect the soil moisture, so as to connect the output port with the input port of ADC module PCF8591 to access to the corresponding digital quantity and transmit the output result to the input port of $\mathrm{CC} 2530$. When using environment temperature sensor 
DS18B20, direct the sensor to the plant, with three pins connected to the ground, power and CC2530 input port respectively.

3. Function Execution Module: it is a small module composed of 3 relays, with the control port of each relay connected to CC2530 input port and sharing $5 \mathrm{~V}$ power with CC2530. 3 relays are connected to the atomization module, full spectrum light and water pump in the water slot to moisten the plant, increase light intensity and pump water from water slot to the pot respectively.

4. Atomization Module: the system adopts ultrasonic ceramic atomization piece to spray the atomized water in the atomization slot from the sprayer to the plant, so as to moisten the plant and decrease environment temperature.

5. Wireless Communication Module: the module is composed of ESP8266 and ZigBee. After the successful settings of gateway, the communications between ESP8266 and ZigBee are carried out via UART, transmitting the bi-directional signals between pot and user mobile phone. During the communication process, due to the potential packet loss or packet sending delay, a handshake protocol is necessary in actual use, in order to ensure reliable communications.

\subsection{Software Framework}

Software of smart pot is composed of cloud, mobile phone and pot.

Cloud: cloud server collects data from pot and database delivers instructions to mobile phone both via MQTT, so as to reduce data transmission, and thus to reduce bandwidth pressure on server. The cloud server layout adopts distributed structure to meet the need of system expansibility and improve error-tolerant rate of the system. Data access layer of cloud is shown in Figure 2.

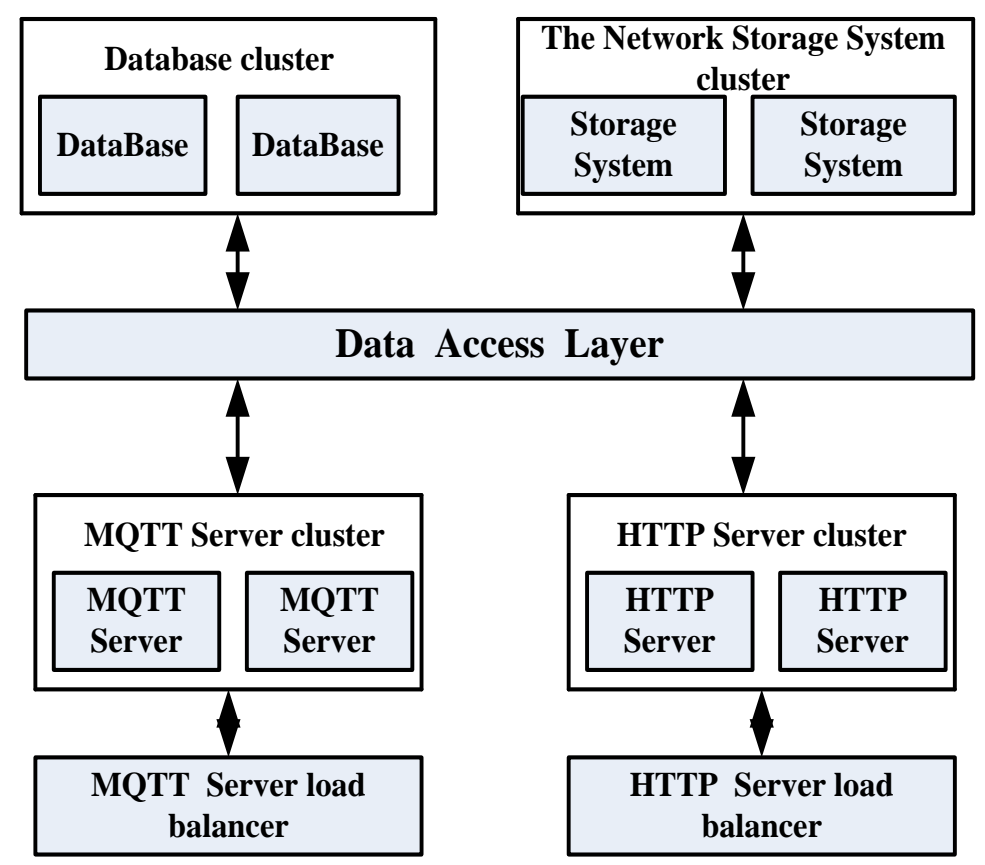

Figure 2. Internal Structure of Distributed Data Access Layer

Mobile Phone: data interaction with cloud via HTTPS mode. ActiveMQ is the most appropriate Message Queue Server which meets distributed structure and supports MQTT and HTTPS, so we choose ActiveMQ as the server for data exchanges in the cloud. The structure of database is shown in Figure 3. 


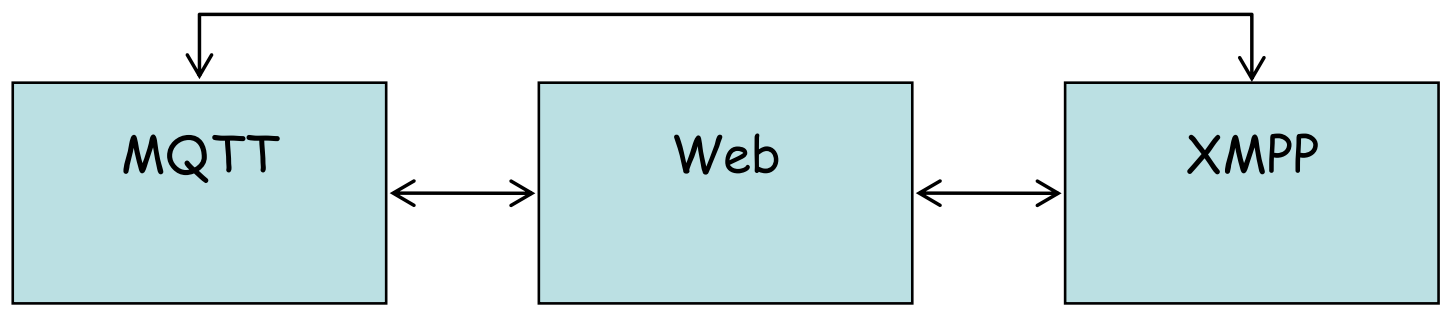

\section{Cluster Plugin}

\section{Authenticate}

\section{Database}

Figure 3. The Structure of Database

Pot: adopting ZigBee protocol stack system.

\section{System Working Principles and Mobile APP Operation Interface}

\subsection{System Working Principles}

Smart pot system has two working modes: hosting and artificial monitoring modes, with structure diagram shown in Figure 3.

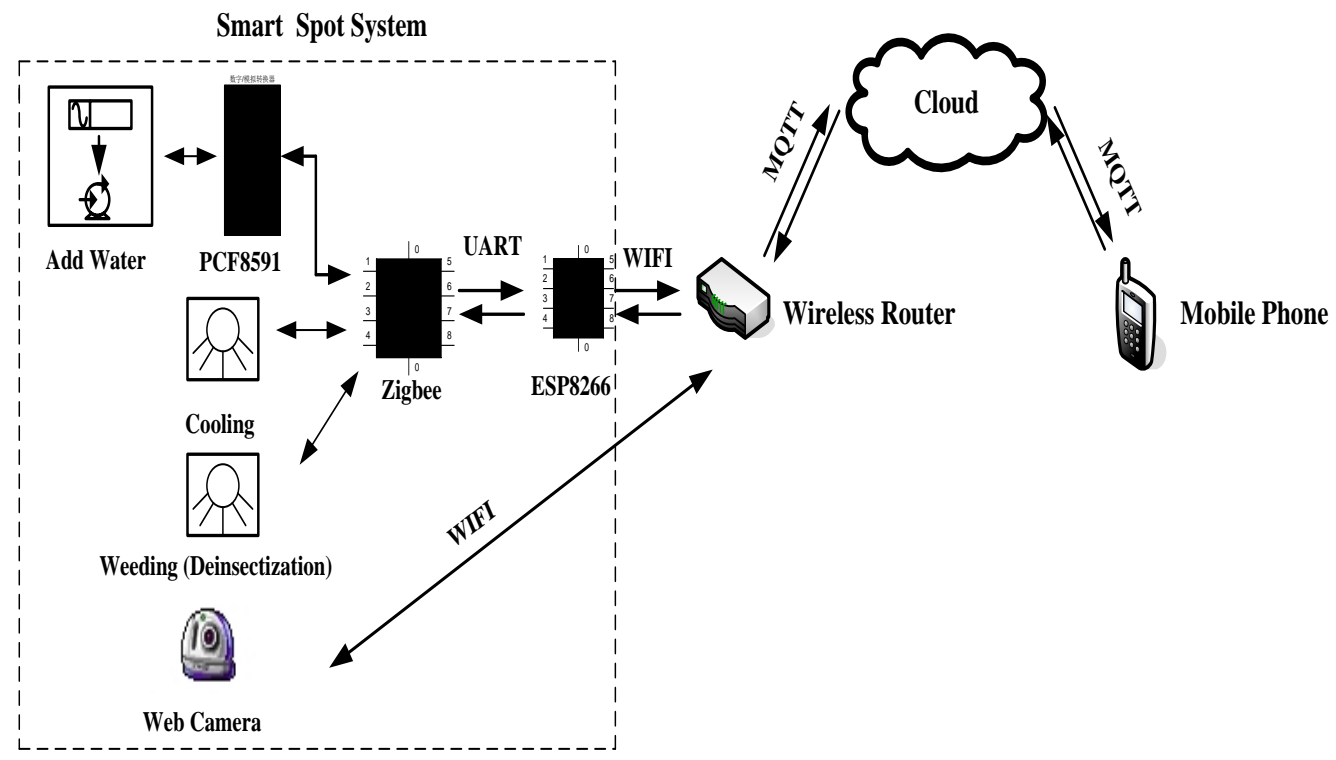

Figure 3. Smart Pot System Structure Diagram

In hosting mode: after turning on their mobile APP and logging in successfully, users click to enter the pot interface to choose hosting, and then the system will automatically add water to the pot, increase moisture (spray mist) and increase light intensity till temperature, moisture and light intensity reach the normal value. As the web camera is 
not allowed to be uploaded in hosting mode, weeding and deinsectization cannot be realized in this mode.

In artificial monitoring mode: after users successfully $\log$ in and select artificial monitoring mode and plant, the system will automatically transmit the current situation (water level, environment temperature and moisture) of the plant to user interface, and complete the above mentioned operations by comparing with the normal value of the breeding status of the plant. Different from hosting mode, users are allowed to open the web camera bound with users to monitor whether there are weeds and injurious insects in the pot, and if any, users can execute weeding and deinsectization. All operation processes are in the following:

In artificial monitoring mode:

Adding water: as shown in Figure 3, after receiving the message from the system, users click corresponding button and the operation instruction will be uploaded to the cloud server via MQTT protocol. When the server receives the instruction, it will transmit it to the node closest to the user home. The node transmits the instruction of adding water via MQTT to home internal wireless router, which will transmit this instruction to ESP8266 of smart pot's wireless network module. ESP8266 is connected to ZigBee via serial port, and transmits this instruction to the serial port of CC2530 after confirmation. The instruction passes ADC of PCF8591 and then goes into the water relay of the execution module, and the relay drives the water pump in the water slot to add up to $200 \mathrm{ml}$ water at a time. After the water is added, the relay will switch off the water pump and at the same time send back a signal of completion to CC2530. The signal will be sent back to the mobile phone through the above process. After user receives the signal and makes confirmation, the process of adding water is completed.

Cooling: as shown in Figure 3, the preceding processes of cooling are basically the same as that of adding water, while the only difference is when ZigBee receives the instruction and transmits it to the execution module, the execution module drives the stepper motor on the bottom of the water slot, and the stepper motor moves to the atomization slot under the instruction to drive the atomization slot to atomize the water in the water slot and then sprays out from the sprayer on the side of the pot.

Weeding (deinsectization): these two functions, which cannot be completed in hosting mode, can be completed only in artificial monitoring mode. After logging in APP, the system will prompt users whether to switch on the web camera related to ID in accordance with the log-in ID. After checking the plant situation, user selects the corresponding APP button if weeding (deinsectization) is necessary. After receiving the instruction, ZigBee transmits it to the execution module, driving the stepper motor on the bottom of the water slot, which moves to the weeding (deinsectization) slot under the instruction to turn on the switch, and the mist sprays out from the sprayer on the side of the pot. During the spraying process, user can adjust the height of the sprayer to the location of the injurious insects.

\subsection{Mobile APP Operation Interface}

Mobile APP operation interface is shown in Figure 4. 


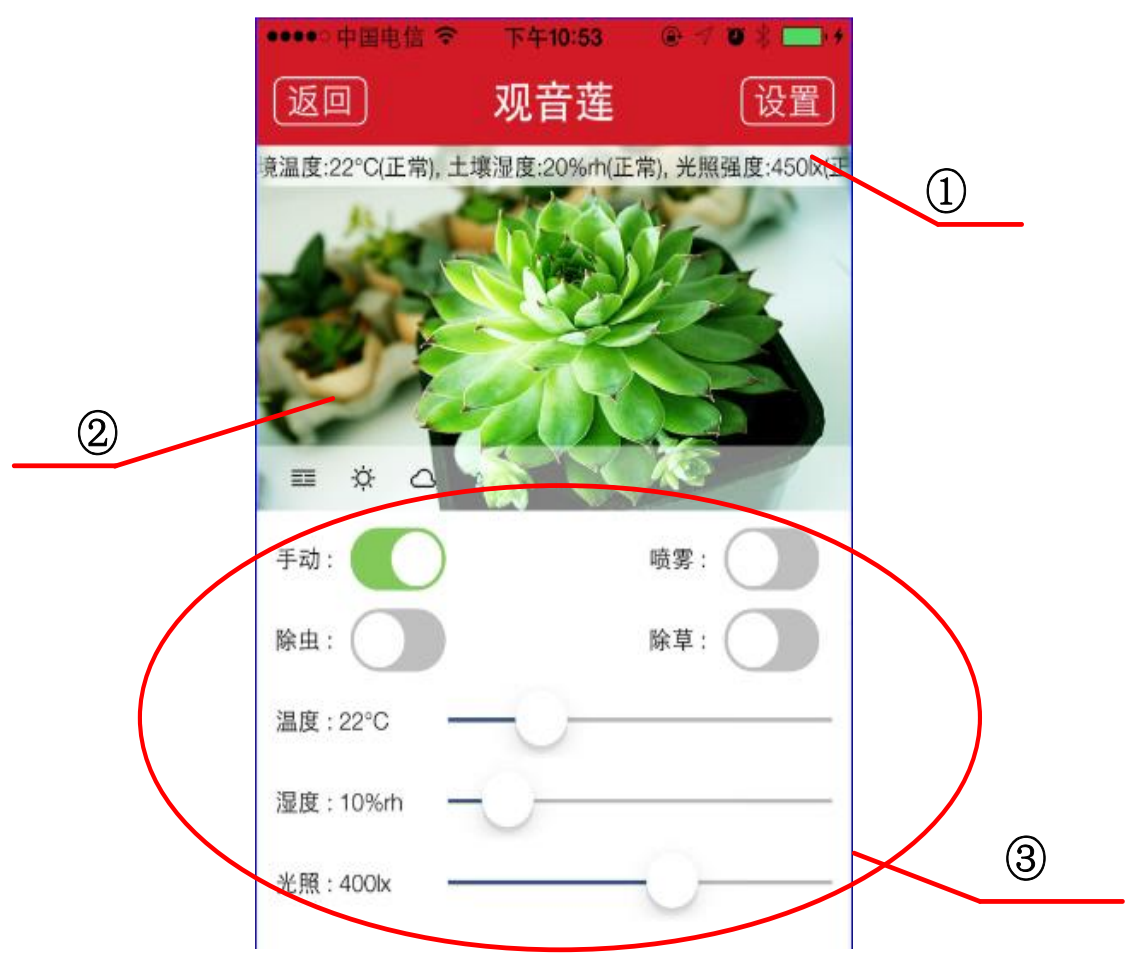

Figure 4. Mobile APP Operation Interface

As shown in Figure, (1) refers to the normal values of all indexes of the plant, which are displayed by scrolling texts. (2) refers to the plant real-time video signal provided by the web camera. After logging in the APP, as long as user clicks in this area, the real-time video signal can be transmitted. and (3) refers to user operation area, consisting of, from top to bottom and from left to right, mode setting button, spraying mist (environment moisture adjustment) button, deinsectization button, weeding button, temperature adjustment process bar, soil moisture adjustment (watering) process bar and light intensity process bar.

\section{Conclusions}

What this paper introduces is a remote smart pot system combining network technology, control technology and Internet of Things, which, compared with the foreign and domestic similar technologies or products, is innovative to some degrees:

1. Different from existing foreign and domestic systems or products of the same kind, it is a real remote wireless smart one with all operations completed through mobile APP.

2. The system has extra functions of executing weeding and deinsectization under the remote instructions via web camera.

The paper points out that user is required to log in mobile APP to verify identity information and the web camera can work only if it is associated with user ID, but it hasn't proposed a more proper solutions regarding user's personal information security, therefore, user's personal information security may be violated during the transmission process, which will be the emphasis in further study.

\section{References}

[1] Z. Zhang, "Study on Home Network Technology Standard and Its Development Strategies in China", Posts and Telecommunications planning, vol. 3, (2004), pp. 3-6.

[2] C. Feng, "Research and Design of Smart Home System Based on Zigbee and ARM9", Master Dissertation, Wuhan University of Technology, vol. 5, (2010), pp. 6-8. 
[3] P. Kinney, "ZigBee technology: Wireless control that simply works", [DB/OL], (2004).

[4] Z. Alliance, "Zigbee Specifications Documents Version 1.0", vol. 12, (2004), pp. 29-150.

[5] V. Handziski, A .Kopke and H. Karl, "A common wireless sensor network architecture", Technical Report TKN-03-012 of the Telecommunications Networks Group, Berlin, (2003).

[6] H. Edgar, J. Callawy and E. H. Callaway, "Wireless sensor networks- Architectures and protocols", Auerbach Publications, New York, vol. 10, (2003), pp. 260-300.

[7] Texas Instruments: CC2530 ZigBee Development Kit User's Guide.

[8] MQ Telemtry Transport.

[9] Lee S, Kim H, Hong D and Ju H, Correlation analysis of MQTT loss and delay according QoS level, Information Network, Bangkok, vol. 8, (2013), pp. 714-717.

\section{Author}

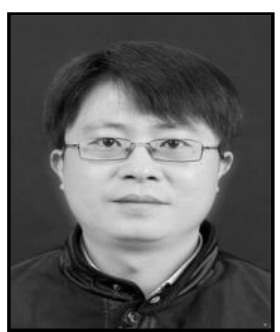

Jijun Xing, he was born in Xi'an City, Shaanxi Province in 1976, has a master's degree in Network Security in Southwest Jiaotong University, and now works in Northwest University of Politics and Law, with major research direction being computer networks and mobile electronic commerce security. 